\title{
An Absolutely Stabilized Finite Element Method for the Stokes Problem
}

\author{
By Jim Douglas, Jr. and Junping Wang \\ Dedicated to Eugene Isaacson on the occasion of his seventieth birthday
}

\begin{abstract}
An absolutely stabilized finite element formulation for the Stokes problem is presented in this paper. This new formulation, which is nonsymmetric but stable without employment of any stability constant, can be regarded as a modification of the formulation proposed recently by Hughes and Franca in [8]. Optimal error estimates in $L^{2}$-norm for the new stabilized finite element approximation of both the velocity and the pressure fields are established, as well as one in $H^{1}$-norm for the velocity field.
\end{abstract}

1. Introduction. We are concerned with finite element methods for the steady state Stokes equation which describes slow motion of an incompressible fluid in $\mathbb{R}^{n}$ with $n \leq 3$. Let $\Omega$ be a bounded domain in $\mathbf{R}^{n}$ and let $u(x)$ and $p(x)$ be the velocity

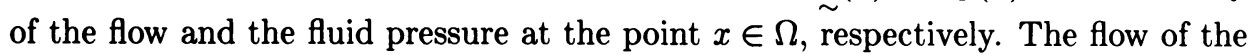
fluid is governed by the Stokes equation

$$
\begin{cases}-\nu \underset{\sim}{u}+\nabla p=f & \text { in } \Omega, \\ \operatorname{div} \underset{\sim}{u}=0 & \text { in } \Omega,\end{cases}
$$

where $\nu$ is the viscosity of the fluid and $f(x)$ the external unit volumetric force acting on the fluid at $x \in \Omega$.

Assume that the velocity of the flow on the boundary $\partial \Omega$ of $\Omega$ is given; i.e.,

$$
\underset{\sim}{u}(x)=\underset{\sim}{g}(x) \quad \text { on } \partial \Omega,
$$

where $\underset{\sim}{g}$ is a function defined on $\partial \Omega$ satisfying the compatibility condition

$$
\int_{\partial \Omega} \underset{\sim}{\sim} \cdot \underset{\sim}{\sim} d s=0
$$

It is not difficult to show that the flow of the fluid is determined uniquely by the Stokes equation (1.1) and the boundary condition (1.2); uniqueness of the pressure field should be understood in the sense of modulo a constant.

For the sake of simplicity, but without loss of generality, we shall take the viscosity $\nu$ to be equal to one and the boundary condition (1.2) to be homogeneous.

Received May 9, 1988.

1980 Mathematics Subject Classification (1985 Revision). Primary 65N30, 65N15, 76D07.

Key words and phrases. Stokes equation, finite element method. 
As usual, a mixed formulation of the problem (1.1) and (1.2) reads as follows: find $(\underset{\sim}{u}, p) \in \underset{\sim}{H_{0}^{1}}(\Omega) \times L_{0}^{2}(\Omega)$ such that

$$
\begin{cases}(\underset{\sim}{\nabla} \underset{\sim}{\nabla} \underset{\sim}{v})-(\operatorname{div} \underset{\sim}{v}, p)=(\underset{\sim}{f}, \underset{\sim}{v}), & \underset{\sim}{v} \in \underset{\sim}{H_{0}^{1}}(\Omega), \\ (\operatorname{div} \underset{\sim}{u}, w)=0, & w \in L_{0}^{2}(\Omega),\end{cases}
$$

where $H^{s}(\Omega)=\left(H^{s}(\Omega)\right)^{n}$, and $H^{s}(\Omega)$ is the usual Sobolev space $W^{s, 2}(\Omega)$ with norm defined by

$$
\|u\|_{s}^{2}=\sum_{|i| \leq s}\left\|\nabla^{i} u\right\|_{0}^{2}, \quad u \in H^{s}(\Omega)
$$

and

$$
\|u\|_{0}^{2}=\int_{\Omega} u^{2} d \Omega, \quad u \in L^{2}(\Omega) .
$$

Let $L_{0}^{2}(\Omega)$ be the subspace of $L^{2}(\Omega)$ consisting of all such functions in $L^{2}(\Omega)$ having mean value zero. Also, let

$$
H_{0}^{1}(\Omega)=\left\{v \in H^{1}(\Omega) ; v=0 \text { on } \partial \Omega\right\} .
$$

The standard mixed finite element method for the Stokes problem in its primitive variables is based on a triangulation, $\Im_{h}$, of $\Omega$ and a finding of a pair of finite element spaces $X^{h} \times M^{h} \subset \underset{\sim}{H_{0}^{1}}(\Omega) \times L_{0}^{2}(\Omega)$ associated with the triangulation $\Im_{h}$ such that

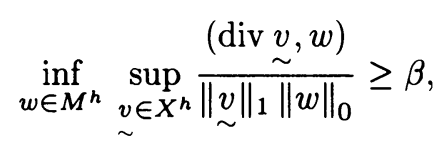

where $\beta$ is a positive constant independent of $h$, the maximum of the diameters of triangles in $\Im_{h}$. The inf-sup condition (1.4) is the so-called Babuska-Brezzi stability condition and plays an important role in the analysis of the stability and the convergence of the mixed finite element method for the Stokes problem (see [1], [2] and [6]). For a general description of the stability condition (1.4), we refer to $[5]$.

It is clear that, owing to the stability condition (1.4), not every combination of finite element spaces $X^{h}$ and $M^{h}$ can be applied to the standard mixed finite element formulation of the Stokes problem to obtain an adequate approximation to the exact solution. As a simple example, let us take

$$
X^{h}=\left\{\underset{\sim}{v}: \underset{\sim}{v} \text { is } C^{0} \text {-piecewise linear }\right\}
$$

and

$$
M^{h}=\{w: w \text { is piecewise constant }\} .
$$

It is not difficult to show that this combination is unacceptable for the standard mixed finite element formulation, although it seems to be the simplest feasible space. In fact, most of the known spaces are not quite natural and, therefore, involve some basis functions that are not found in many of the engineering code packages which are commonly used.

Recently, Hughes, Franca, and Balestra [7] proposed a stabilized finite element formulation for the Stokes problem for which the stability constraint (1.4) is not needed. Therefore, more natural and simpler finite element spaces can be used. A 
more complete analysis of the method of Hughes, Franca, and Balestra has been given by Brezzi and Douglas [3].

The idea of Hughes, Franca, and Balestra in [7] can be interpreted as follows: let $\Im_{h}$ be a quasi-regular triangulation of $\Omega$, and let $X^{h}$ and $M^{h}$ be two finite element spaces satisfying

$$
X^{h} \subset \underset{\sim}{H} H_{0}^{1}(\Omega)
$$

and

$$
M^{h} \subset H^{1}(\Omega) \cap L_{0}^{2}(\Omega) .
$$

A mesh-dependent norm on $X^{h} \times M^{h}$ can be defined in the following way:

$$
\|(\underset{\sim}{v}, w)\|^{2}=\|\underset{\sim}{v}\|_{0}^{2}+\sum_{T} h_{T}^{2}\|\nabla w\|_{0, T}^{2}
$$

where $h_{T}=\operatorname{diam}(T)$ for $T \in \Im_{h}$.

The stabilized finite element approximation $\left(\underset{\sim}{u^{h}}, p^{h}\right)$ is given by solving the following linear system:

$$
\begin{cases}\left(\underset{\sim}{\nabla} u^{h}, \nabla \underset{\sim}{v}\right)-\left(\operatorname{div} \underset{\sim}{v}, p^{h}\right)=(\underset{\sim}{f}, \underset{\sim}{v}), & \underset{\sim}{v} \in X^{h}, \\ \left(\underset{\sim}{\operatorname{div}}{\underset{\sim}{u}}^{h}, w\right)+\alpha \sum_{T} h_{T}^{2}\left(\nabla p^{h}-\Delta{\underset{\sim}{u}}^{h}, \nabla w\right)_{T}=\alpha \sum_{T} h_{T}^{2}(\underset{\sim}{f}, \nabla w)_{T}, & w \in M^{h},\end{cases}
$$

where $\alpha$ is a positive number. The term on the left containing the constant $\alpha$ adds stability to the standard mixed finite element formulation for the Stokes problem and thus plays an important role for the stability and convergence of the formulation (1.6). The $\alpha$-term on the right maintains consistency.

In [7], Hughes et al. proved that there exists a constant $\alpha_{0}>0$ depending only on the shapes of the triangles $T \in \Im_{h}$ such that the linear system (1.6) has a unique solution $\left({\underset{\sim}{u}}^{h}, p^{h}\right)$ for any $\alpha \in\left(0, \alpha_{0}\right)$ and that the solution $\left(\underset{\sim}{u^{h}}, p^{h}\right)$ converges to the exact solution $(\underset{\sim}{u}, p)$ of the Stokes problem in the mesh-dependent norm (1.5), as $h \rightarrow 0$, if $\alpha$ is bounded away from zero. An error estimate in the $L^{2}$-norm for the stabilized finite element scheme (1.6) has been established by Brezzi and Douglas in [3], where they also presented a modification of (1.6).

The stabilized formulation (1.6) requires continuity for the pressure interpolation; this requirement has been proved to be removable by Hughes and Franca in [8] by introducing a jump operator into the formulation (1.6). Actually, the stabilized finite element formulation given in [8] developed (1.6) in a more sophisticated fashion; it is not only symmetric but also suitable for any combination of finite element spaces $X^{h}$ and $M^{h}$, either continuous or discontinuous for the pressure component. Nevertheless, the solvability and the convergence of the stabilized finite element formulation of Hughes and Franca still rely on a stability constant $\alpha$ which is shape-dependent. An error estimate in a mesh-dependent norm analogous to (1.5) was derived by Hughes and Franca in [8].

In this paper we shall propose an absolutely stabilized finite element formulation for the Stokes problem, which can be viewed as a modification of the formulation of Hughes and Franca [8], although the forthcoming formulation was discovered by the authors independently of [8]. As we shall see in the next section, the modified formulation is stable and convergent without the employment of a stability constant. 
Error estimates in the $L^{2}$-norm for both the velocity and the pressure are derived under an assumption of shape regularity on the triangulation $\Im_{h}$, as well as one in the $H^{1}$-norm for the velocity field.

The paper is organized in the following way. Our finite element formulation will be presented in Section 2. Then in Section 3 we establish error estimates for this finite element approximation.

2. Finite Element Formulation. The aim of this section is to present an absolutely stabilized finite element formulation for the Stokes problem. For the sake of convenience of argument, we shall assume that $\Omega$ is a polygonal domain in $\mathbf{R}^{n}$. However, an extension to more general domains can be made without any difficulty.

Let $\Im_{h}=\{T\}$ be a triangulation of $\Omega$. Let $\mathscr{R}_{h}$ be a polygonalization of $\Omega$ such that $\Im_{h}$ is a refinement of $\mathscr{R}_{h}$. A particular example for $\mathscr{R}_{h}$ is a copy of $\Im_{h}$. Let $\Gamma^{h}$ be the collection of the edges of all $R$ belonging to $\mathscr{R}_{h} ; \Gamma^{h}$ can be decomposed into two parts: boundary edges $\Gamma_{1}^{h}$ and interior edges $\Gamma_{0}^{h}$. More specifically, let

$$
\Gamma_{1}^{h}=\left\{e \subset \partial \Omega: e \text { is an edge of some } R \in \mathscr{R}_{h}\right\}
$$

and

$$
\Gamma_{0}^{h}=\Gamma^{h} \backslash \Gamma_{1}^{h} .
$$

Denote by $h_{T}=\operatorname{diam}(T)$ and $h_{e}=\operatorname{diam}(e)$ the diameters of $T \in \Im_{h} \cup \mathscr{R}_{h}$ and $e \in \Gamma^{h}$, respectively. Set $h=\max _{T}\left\{h_{T}\right\}$ and assume that $\Omega=\Omega^{h}=\bigcup_{R \in \mathscr{R}_{h}} R$. Let

$$
H_{h}^{k}=\left\{v \in L^{2}\left(\Omega^{h}\right):\left.\quad v\right|_{R} \in H^{k}(R), R \in \mathscr{R}_{h}\right\} .
$$

Let $e \in \Gamma_{0}^{h}$, and let $e=R_{1} \cap R_{2}$. The jump of $w \in H_{h}^{1}$ across $e$ is given by

$$
\llbracket w \rrbracket=t r_{e, R_{1}}(w)-t r_{e, R_{2}}(w) ;
$$

interchanging $R_{1}$ and $R_{2}$ will have no effect on the finite element procedure.

Let $X^{h}$ and $M^{h}$ be the two finite element spaces associated with $\Im_{h}$ and $\mathscr{R}_{h}$, respectively, defined as follows:

$$
\begin{gathered}
X^{h}=\left\{\underset{\sim}{v} \in \underset{\sim}{H_{0}^{1}}(\Omega):\left.\underset{\sim}{v}\right|_{T} \in{\underset{\sim}{P}}^{k}(T), T \in \Im_{h}\right\}, \\
M^{h}=\left\{w \in L_{0}^{2}(\Omega):\left.w\right|_{R} \in P^{l-1}(R), R \in \mathscr{R}_{h}\right\},
\end{gathered}
$$

where $k$ and $l$ are two positive integers and $P^{s}(T)$ indicates the collection of polynomials of degree not greater than $s$. The finite element spaces $X^{h}$ and $M^{h}$ are quite simple and natural and are easily constructed. It is these spaces that we are going to use to approximate the Stokes problem. In what follows, we shall take $l=k$ in (2.3), although $l$ can be taken to be independent of $k$.

We define a mesh-dependent norm on $X^{h} \times M^{h}$ as follows:

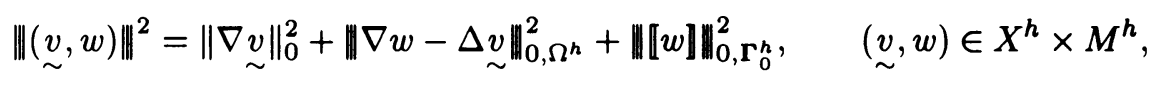

where

$$
\|\nabla w-\Delta \underset{\sim}{v}\|_{0, \Omega^{h}}^{2}=\alpha \sum_{T} h_{T}^{2}\|\nabla w-\Delta \underset{\sim}{v}\|_{0, T}^{2}
$$


and

$$
\|\llbracket w \rrbracket\|_{0, \Gamma_{0}^{h}}^{2}=\beta \sum_{e \in \Gamma_{0}^{h}} h_{e}(\llbracket w \rrbracket, \llbracket w \rrbracket)_{e} .
$$

Here, $(\cdot, \cdot)_{D}$ indicates the inner product in $L^{2}(D)$ and $(\cdot, \cdot)$ will be that in $L^{2}(\Omega)$. The positive constants $\alpha$ and $\beta$ in (2.5) and (2.6) are arbitrary and do not depend on the triangulation $\Im_{h}$. It is clear that $(2.4)$ is well defined for $(\underset{\sim}{v}, w)$ belonging to $\left(\underset{\sim}{H_{0}^{1}}(\Omega) \cap \underset{\sim}{H_{h}^{2}}\right) \times H_{h}^{1}$.

LEMMA 2.1. The relation (2.4) defines a norm over $X^{h} \times M^{h}$.

Proof. It suffices to check that $\|(\underset{\sim}{v}, w)\|=0$ implies $\underset{\sim}{v}=0$ and $w=0$. In fact, $\|(\underset{\sim}{v}, w)\|=0$ implies $\underset{\sim}{v}=0, \llbracket w \rrbracket=0$, and $w=$ constant on each $T \in \Im_{h}$. Thus, it follows from the fact $w \in L_{0}^{2}(\Omega)$ that $w=0$.

We are ready now to present a new stabilized finite element formulation for the Stokes problem. Let us begin with a bilinear form $\Phi$ defined on $X^{h} \times M^{h}$ by

$$
\begin{aligned}
\underset{\sim}{\boldsymbol{\Phi}}((q, \chi),(\underset{\sim}{v}, w))= & (\underset{\sim}{\nabla}, \underset{\sim}{\nabla v})-(\operatorname{div} \underset{\sim}{v}, \chi)+(\operatorname{div} \underset{\sim}{q}, w) \\
& +\alpha \sum_{T} h_{T}^{2}(\nabla \chi-\Delta \underset{\sim}{q}, \nabla w-\Delta \underset{\sim}{v})_{T} \\
& +\beta \sum_{e \in \Gamma_{0}^{h}} h_{e}(\llbracket \chi \rrbracket, \llbracket w \rrbracket)_{e},
\end{aligned}
$$

where $\alpha$ and $\beta$ are the same constants as in (2.5) and (2.6). The bilinear form (2.7) is coercive on $X^{h} \times M^{h}$, since

$$
\boldsymbol{\Phi}((\underset{\sim}{v}, w),(\underset{\sim}{v}, w))=\|(\underset{\sim}{v}, w)\|^{2}, \quad(\underset{\sim}{v}, w) \in X^{h} \times M^{h}
$$

Our absolutely stabilized finite element approximation for the Stokes problem consists in finding $\left(\underset{\sim}{u^{h}}, p^{h}\right) \in X^{h} \times M^{h}$ such that

$$
\begin{aligned}
& \boldsymbol{\Phi}\left(\left({\underset{\sim}{u}}^{h}, p^{h}\right),(\underset{\sim}{v}, w)\right) \\
& \quad=(\underset{\sim}{f}, \underset{\sim}{v})+\alpha \sum_{T} h_{T}^{2}(\underset{\sim}{f}, \nabla w-\Delta \underset{\sim}{v})_{T}, \quad(\underset{\sim}{v}, w) \in X^{h} \times M^{h} .
\end{aligned}
$$

It follows from the coercivity of the bilinear form $\Phi$ that the linear system (2.9) is uniquely solvable. The bilinear form (2.7) differs from that of [8] in having the sign of the second divergence term to be positive, so that it corresponds a bit more closely with the usual bilinear form associated with (1.3). We call (2.9) an absolutely stabilized finite element formulation for the Stokes problem since no constraints other than positivity need be imposed on $\alpha$ and $\beta$.

THEOREM 2.1. There exists a unique solution $\left.\underset{\sim}{u^{h}}, p^{h}\right)$ in $X^{h} \times M^{h}$ of (2.9). 
The linear system (2.9) can be decomposed into a coupled system by separating the effects of $\underset{\sim}{v}$ and $w$. The resulting equations are given by

$$
\left\{\begin{array}{c}
\left(\underset{\sim}{\nabla} u_{\sim}^{h}, \nabla \underset{\sim}{v}\right)-\left(\underset{\sim}{\operatorname{div}}, p^{h}\right)-\alpha \sum_{T} h_{T}^{2}\left(\nabla p^{h}-\Delta \underset{\sim}{u^{h}}, \Delta \underset{\sim}{v}\right)_{T} \\
=(\underset{\sim}{f}, \underset{\sim}{v})-\alpha \sum_{T} h_{T}^{2}(\underset{\sim}{f}, \Delta \underset{\sim}{v})_{T}, \quad \underset{\sim}{v} \in X^{h}, \\
\left(\underset{\sim}{\left.\operatorname{div} \underset{\sim}{u^{h}}, w\right)}+\alpha \sum_{T} h_{T}^{2}(\nabla p^{h}-\Delta \underbrace{h}, \nabla w)_{T}+\beta \sum_{e \in \Gamma_{0}^{h}} h_{e}\left(\llbracket p^{h} \rrbracket, \llbracket w \rrbracket\right)_{e}\right. \\
=\alpha \sum_{T} h_{T}^{2}(\underset{\sim}{f}, \nabla w)_{T}, \quad w \in M^{h} .
\end{array}\right.
$$

Let $\underset{\sim}{u}, p)$ be the unique solution of the Stokes problem (1.1) and (1.2). Formally, we have

$$
\left.\alpha \sum_{T} h_{T}^{2}(\nabla p-\Delta \underset{\sim}{u}, \underset{\sim}{v})_{T}=\alpha \sum_{T} h_{T}^{2} \underset{\sim}{f}, \underset{\sim}{v}\right)_{T}, \quad \underset{\sim}{v} \in X^{h}
$$

and

$$
\alpha \sum_{T} h_{T}^{2}(\nabla p-\Delta \underset{\sim}{u}, \nabla w)_{T}=\alpha \sum_{T} h_{T}^{2}(\underset{\sim}{f}, \nabla w)_{T}, \quad w \in M^{h} .
$$

Thus, combining (1.3) with the above two equalities gives

$$
\begin{aligned}
&(\underset{\sim}{u}, \nabla \underset{\sim}{v})-(\underset{\sim}{\operatorname{div}} \underset{\sim}{v} p)-\alpha \sum_{T} h_{T}^{2}(\nabla p-\Delta \underset{\sim}{u}, \Delta \underset{\sim}{v})_{T} \\
&=(\underset{\sim}{f} \underset{\sim}{v})-\alpha \sum_{T} h_{T}^{2}(\underset{\sim}{f}, \Delta \underset{\sim}{v})_{T}, \quad \underset{\sim}{v} \in X^{h}
\end{aligned}
$$

and

$$
\left.(\operatorname{div} \underset{\sim}{u}, w)+\alpha \sum_{T} h_{T}^{2}(\nabla p-\Delta \underset{\sim}{u}, \nabla w)_{T}=\alpha \sum_{T} h_{T}^{2} \underset{\sim}{f}, \nabla w\right)_{T}, \quad w \in M^{h} .
$$

Adding (2.13) to (2.14) gives

$$
\boldsymbol{\Phi}((\underset{\sim}{u}, p),(\underset{\sim}{v}, w))=(\underset{\sim}{f}, \underset{\sim}{v})+\alpha \sum_{T} h_{T}^{2}(\underset{\sim}{f}, \nabla w-\Delta \underset{\sim}{v})_{T}, \quad(\underset{\sim}{v}, w) \in X^{h} \times M^{h}
$$

where $\llbracket p \rrbracket$ should be understood to be zero on $\Gamma_{0}^{h}$. In fact, this relation does hold for smooth $p$, for instance $p \in H^{1}$. Thus, (2.15) and (2.9) imply the following error equation:

$$
\boldsymbol{\Phi}\left(\left(\left(\underset{\sim}{u}-\underset{\sim}{u^{h}}\right),\left(p-p^{h}\right)\right),(\underset{\sim}{v}, w)\right)=0, \quad(\underset{\sim}{v}, w) \in X^{h} \times M^{h} .
$$

In the important special case that the finite element space for $k=l=1$ is used to approximate the Stokes problem via the formulation (2.9), the bilinear form $\boldsymbol{\Phi}$ reduces to

$$
\boldsymbol{\Phi}_{1}((\underset{\sim}{q}, \chi),(\underset{\sim}{v}, w))=(\underset{\sim}{q}, \underset{\sim}{\nabla})-(\operatorname{div} \underset{\sim}{v}, \chi)+(\operatorname{div} \underset{\sim}{q}, w)+\beta \sum_{e \in \Gamma_{0}^{h}} h_{e}(\llbracket \chi \chi \rrbracket, \llbracket w \rrbracket)_{e}
$$

and the corresponding finite-dimensional linear system becomes

$$
\Phi_{1}\left(\left({\underset{\sim}{u}}^{h}, p^{h}\right),(\underset{\sim}{v}, w)\right)=(\underset{\sim}{f}, \underset{\sim}{v}), \quad(\underset{\sim}{v}, w) \in X^{h} \times M^{h} .
$$


Clearly, $\boldsymbol{\Phi}_{1}$ involves neither second-order derivatives over the velocity field nor any derivatives over the pressure field. Also, it is easy to see that $\underset{\sim}{u}, p)$ satisfies the equation (2.18). Thus, we have an analogue of (2.16) for $\boldsymbol{\Phi}_{1}$. This observation should be understood in the remainder of this paper. In this case, our method coincides with that of Hughes and Franca [8].

For the sake of simplicity of analysis, we shall take $\alpha=\beta=1$ and $\mathscr{R}_{h}=\Im_{h}$ throughout the end of this paper; it is easy to extend the analysis to the general case. The finite element spaces $X^{h}$ and $M^{h}$ can be associated with different triangulations of $\Omega$. This could be important from the computational point of view, for a significant reduction of the number of degrees of freedom for $M^{h}$ is possible if $\mathscr{R}_{h} \neq \Im_{h}$. The precise effect of such a choice is not completely clear from the error estimates that follow.

3. Error Analysis. We shall assume $\underset{\sim}{(u, p)}$ to be the exact solution of the Stokes problem (1.1) and (1.2). Let $\left(\underset{\sim}{u}{ }^{h}, p^{h}\right)$ be the stabilized finite element approximation of the Stokes problem obtained by solving the linear system (2.9). Our primary goal is to establish optimal error estimates in the $L^{2}$-norm for the velocity and the pressure fields, following an optimal error estimate in the $H^{1}$-norm for the velocity field.

Let us assume the following approximation properties for the finite element spaces $X^{h}$ and $M^{h}$ : for any $(\psi, \varphi) \in \underset{\sim}{H_{0}^{1}}(\Omega) \times L_{0}^{2}(\Omega)$, there exists an interpolation of $\underset{\sim}{(\psi, \varphi)}$, denoted by $\left(\underset{\sim}{\left.\psi^{I}, \tilde{\varphi}^{I}\right)}\right.$, such that

$$
\begin{aligned}
& \| \underset{\sim}{\|} \underset{\sim}{\psi} \underset{\sim}{I}) \|_{0}+\left(\sum_{T} h_{T}^{-2}\left\|\underset{\sim}{\psi} \underset{\sim}{\psi^{I}}\right\|_{0, T}^{2}\right)^{1 / 2}+\left(\sum_{e \in \Gamma_{0}^{h}} h_{e}^{-1}\left\|\left(\underset{\sim}{\psi} \underset{\sim}{\psi^{I}}\right) \cdot \underset{\sim}{n}\right\|_{0, e}^{2}\right)^{1 / 2} \\
& \leq C h^{m-1}\|\underset{\sim}{\psi}\|_{m} \quad \text { if } \underset{\sim}{\psi \in} \underset{\sim}{H^{r}}(\Omega),
\end{aligned}
$$

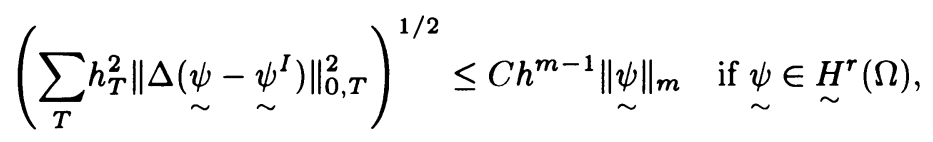

where $m=\min (k+1, r)$, and

$$
\left\|\varphi-\varphi^{I}\right\|_{0}+\left\|\llbracket \varphi^{I} \rrbracket\right\|_{0, \Gamma_{0}^{h}} \leq C h^{l}\|\varphi\|_{l} \quad \text { if } \underset{\sim}{\varphi} \in H^{s}(\Omega) \text { and } s \geq 1,
$$

where $l=\min (k, s)$. The symbol $C$ is used to denote a generic constant independent of $h$ and the function being approximated. It is easy to verify that, if the triangulation $\Im_{h}$ is quasi-regular, then (3.1), (3.2) and (3.3) hold for the spaces $X^{h}$ and $M^{h}$ discussed in the previous section.

Let

$$
\begin{array}{ccc}
\underset{\sim}{\xi}={\underset{\sim}{u}}^{h}-{\underset{\sim}{u}}^{I}, & \underset{\sim}{\varsigma}=\underset{\sim}{u}-{\underset{\sim}{u}}^{I}, & {\underset{\sim}{\sim}}_{\sim}=\underset{\sim}{u}-{\underset{\sim}{\sim}}^{h}, \\
\tau=p^{h}-p^{I}, & \eta=p-p^{I}, & e_{p}=p-p^{h} .
\end{array}
$$

By the error equation (2.16),

$$
\boldsymbol{\Phi}((\underset{\sim}{\xi}, \tau),(\underset{\sim}{v}, w))=\boldsymbol{\Phi}((\underset{\sim}{\zeta}, \eta),(\underset{\sim}{v}, w)), \quad(\underset{\sim}{v}, w) \in X^{h} \times M^{h} .
$$


In particular, by setting $(\underset{\sim}{v}, w)=\underset{\sim}{\xi}, \tau)$, we see that

$$
\left.\left.\|(\underset{\sim}{\xi}, \tau)\|^{2}=\underset{\sim}{\boldsymbol{\Phi}}((\underset{\sim}{\xi}, \tau), \underset{\sim}{\xi}, \tau)\right)=\underset{\sim}{\boldsymbol{\Phi}}((\underset{\sim}{\zeta}, \eta), \underset{\sim}{\xi}, \tau)\right)
$$

Before going to the error estimate, we should like to define shape regularity for the triangulation $\Im_{h}$ : $\Im_{h}$ is shape regular if the ratio of the diameter of the circumscribed ball for $T \in \Im_{h}$ to that of the inscribed ball is bounded, independently of $T \in \Im_{h}$.

LEMMA 3.1. Let the triangulation $\Im_{h}$ be shape regular and assume that $\underset{\sim}{q} \in$ $H_{0}^{1}(\Omega)$. Then, for any $\varepsilon>0$, there exists a constant $C(\varepsilon)$ depending only on $\varepsilon$ and the shape regularity of $\Im_{h}$ such that

$$
\begin{aligned}
|(\operatorname{div} \underset{\sim}{q}, w)| \leq \varepsilon\|(\underset{\sim}{v}, w)\|^{2} & \\
& +C(\varepsilon)\left(\sum_{T} h_{T}^{-2}\|\underset{\sim}{q}\|_{0, T}^{2}+\left.\sum_{e \in \Gamma_{0}^{h}} h_{e}^{-1} \int_{e} \underset{\sim}{\mid q} \underset{\sim}{n_{e}}\right|^{2} d s\right), \\
& (\underset{\sim}{v}, w) \in X^{h} \times M^{h} .
\end{aligned}
$$

Proof. Integration by parts gives

$$
\left.(\operatorname{div} \underset{\sim}{q}, w)=\sum_{T}(\underset{\sim}{q},-\nabla w)_{T}+\sum_{e \in \Gamma_{0}^{h}} \underset{\sim}{q} \cdot \underset{\sim}{n_{e}}, \llbracket w \rrbracket\right)_{e},
$$

where $\underset{\sim}{n_{e}}$ is a normal vector for $e$. Since

$$
(\underset{\sim}{q},-\nabla w)_{T}=(\underset{\sim}{q},-\nabla w+\Delta \underset{\sim}{v})_{T}-(\underset{\sim}{q}, \Delta \underset{\sim}{v})_{T}
$$

we have

$$
\begin{aligned}
|(\operatorname{div} \underset{\sim}{q}, w)| \leq & \sum_{T}\left|(\underset{\sim}{q}, \underset{\sim}{v}-\nabla w)_{T}\right|+\sum_{T}\left|(\underset{\sim}{q}, \Delta \underset{\sim}{v})_{T}\right| \\
& +\sum_{e \in \Gamma_{0}^{h}}\left|\left(\underset{\sim}{q} \cdot \underset{\sim}{n_{e}}, \llbracket w \rrbracket\right)_{e}\right| .
\end{aligned}
$$

Thus, for any $\varepsilon>0, \delta>0$,

$$
\begin{aligned}
|(\operatorname{div} \underset{\sim}{q}, w)| \leq & \frac{1}{4 \varepsilon} \sum_{T} h_{T}^{-2}\|\underset{\sim}{q}\|_{0, T}^{2}+\varepsilon \sum_{T} h_{T}^{2}\|\nabla w-\Delta \underset{\sim}{v}\|_{0, T}^{2} \\
& +\delta \sum_{T} h_{T}^{2}\|\Delta \underset{\sim}{v}\|_{0, T}^{2}+\underset{4}{\frac{1}{4 \delta}} \sum_{T} h_{T}^{-2}\|\underset{\sim}{q}\|_{0, T}^{2} \\
& +\left.\frac{1}{4 \varepsilon} \sum_{e \in \Gamma_{0}^{h}} h_{e}^{-1} \int_{e} \underset{\sim}{q} \cdot \underset{\sim}{n_{e}}\right|^{2} d s+\varepsilon\|\| w\|\|_{0, \Gamma_{0}^{h}}^{2} .
\end{aligned}
$$

Note that the shape regularity assumption on $\Im_{h}$ implies that there exists a constant $Q$ independent of $h_{T}$ such that

$$
\sum_{T} h_{T}^{2}\|\Delta \underset{\sim}{v}\|_{0, T}^{2} \leq Q\|\nabla \underset{\sim}{v}\|_{0}^{2}, \quad \underset{\sim}{v} \in X^{h}
$$


Thus, by taking $\delta=\varepsilon Q^{-1}$,

$$
\begin{aligned}
|(\operatorname{div} \underset{\sim}{q}, w)| \leq \varepsilon & \| \underset{\sim}{v}, w) \|^{2} \\
& +C(\varepsilon)\left(\sum_{T} h_{T}^{-2}\|\underset{\sim}{q}\|_{0, T}^{2}+\sum_{e \in \Gamma_{0}^{h}} h_{e}^{-1} \int_{e}\left|\underset{\sim}{q} \cdot \underset{\sim}{n_{e}}\right|^{2} d s\right),
\end{aligned}
$$

where $C(\varepsilon)=(Q+1)(4 \varepsilon)^{-1}$. $\square$

Let us now derive the following basic error estimate for the stabilized finite element approximation.

THEOREM 3.1. Assume that $\Im_{h}$ is shape regular. Then,

$$
\begin{aligned}
& \left\|\left(\underset{\sim}{e_{u}}, e_{p}\right)\right\| \leq 3\left(\|(\underset{\sim}{s}, \eta)\|+\|\eta\|_{0}\right) \\
& +C\left(\sum_{T} h_{T}^{-2}\|\underset{\sim}{s}\|_{0, T}^{2}+\left.\sum_{e \in \Gamma_{0}^{h}} h_{e}^{-1} \int_{e} \underset{\sim}{\mid \zeta} \cdot \underset{\sim}{n_{e}}\right|^{2} d s\right)^{1 / 2} .
\end{aligned}
$$

Moreover,

$$
\left\|\left(\underset{\sim}{e_{u}}, e_{p}\right)\right\| \leq C h^{k}\left(\|\underset{\sim}{u}\|_{k+1}+\|p\|_{k}\right)
$$

and

$$
\left\|\nabla\left(\underset{\sim}{u}-\underset{\sim}{u^{h}}\right)\right\|_{0} \leq C h^{k}\left(\|\underset{\sim}{u}\|_{k+1}+\|p\|_{k}\right),
$$

provided that $\underset{\sim}{u}, p) \in{\underset{\sim}{H}}^{k+1}(\Omega) \times H^{k}(\Omega)$.

Proof. By (3.5),

$$
\begin{aligned}
\|(\underset{\sim}{\xi}, \tau)\|^{2}= & \underset{\sim}{\boldsymbol{\Phi}}(\underset{\sim}{(\zeta, \eta)}, \underset{\sim}{\xi}, \tau)) \\
= & (\underset{\sim}{\nabla}, \underset{\sim}{\nabla})-(\operatorname{div} \underset{\sim}{\xi}, \eta)+(\underset{\sim}{\operatorname{div}} \underset{\sim}{\zeta}, \tau) \\
& \left.+\sum_{T} h_{T}^{2}(\nabla \eta-\Delta \underset{\sim}{\zeta}, \nabla \tau-\Delta \underset{\sim}{\xi})_{T}+\sum_{e \in \Gamma_{0}^{h}} h_{e}([\eta\rceil],[\tau]\right)_{e} .
\end{aligned}
$$

Thus,

$$
\begin{aligned}
& \|(\underset{\sim}{\xi}, \tau)\|^{2} \leq\left(\|\nabla \underset{\sim}{s}\|_{0}+\|\eta\|_{0}\right)\|\nabla \underset{\sim}{\xi}\|_{0}+|(\operatorname{div} \underset{\sim}{s}, \tau)| \\
& +\sum_{T} h_{T}^{2}\|\nabla \eta-\underset{\sim}{s}\|_{0, T}\|\nabla \tau-\Delta \underset{\sim}{\xi}\|_{0, T} \\
& +\sum_{e \in \Gamma_{0}^{h}} h_{e}\|\llbracket \eta \rrbracket\|_{0, e}\|\llbracket \tau \rrbracket\|_{0, e} \\
& \left.\leq \frac{1}{4}\left(\underset{\sim}{\|\underset{\xi}{\xi}\|_{0}^{2}}+\sum_{T} h_{T}^{2}\left\|\nabla \tau-\underset{\sim}{\underline{\xi} \|_{0, T}^{2}}+\sum_{e \in \Gamma_{0}^{h}} h_{e}\right\| \llbracket \tau\right] \|_{0, e}^{2}\right) \\
& +2\|\nabla \underset{\sim}{\zeta}\|_{0}^{2}+2\|\eta\|_{0}^{2}+\sum_{T} h_{T}^{2}\|\nabla \eta-\Delta \underset{\sim}{\varsigma}\|_{0, T}^{2} \\
& +\sum_{e \in \Gamma_{0}^{h}} h_{e}\|\| \eta\|\|_{0, e}^{2}+|(\operatorname{div} \underset{\sim}{S}, \tau)| \\
& \leq \frac{1}{4}\|(\underset{\sim}{\xi}, \tau)\|^{2}+2\|(\underset{\sim}{s}, \eta)\|^{2}+2\|\eta\|_{0}^{2}+|(\operatorname{div} \underset{\sim}{s}, \tau)| .
\end{aligned}
$$


To deal with $|(\operatorname{div} \underset{\sim}{s}, \tau)|$ in $(3.15)$, we make use of Lemma 3.1 with $\underset{\sim}{q}$ and $\underset{\sim}{v}, w)$ replaced by $\varsigma$ and $(\tilde{\xi}, \tau)$, respectively. Hence, by taking $\varepsilon=\frac{1}{4}$ in $(3.6)$,

$$
\begin{aligned}
& \left.\|(\underset{\sim}{\xi}, \tau)\|^{2} \leq \frac{1}{2}\|(\underset{\sim}{\xi}, \tau)\|^{2}+2\|\eta\|_{0}^{2}+2 \| \underset{\sim}{(\varsigma}, \eta\right) \|^{2} \\
& +C\left(\sum_{T} h_{T}^{-2}\|\underset{\sim}{\zeta}\|_{0, T}^{2}+\sum_{e \in \Gamma_{0}^{h}} h_{e}^{-1}\left\|\underset{\sim}{S} \cdot \underset{\sim}{n_{e}}\right\|_{0, e}^{2}\right) .
\end{aligned}
$$

Thus, we are led to the following inequality:

$$
\begin{aligned}
\|(\underset{\sim}{\xi}, \tau)\| \leq & 2\left(\underset{\sim}{(s, \eta) \|}+\|\eta\|_{0}\right) \\
& +C\left(\sum_{T} h_{T}^{-2}\|\underset{\sim}{S}\|_{0, T}^{2}+\sum_{e \in \Gamma_{0}^{h}} h_{e}^{-1}\left\|\underset{\sim}{S} \cdot \underset{\sim}{n_{e}}\right\|_{0, e}^{2}\right)^{1 / 2} .
\end{aligned}
$$

Now, combining (3.16) with the usual triangle inequality gives (3.11) immediately. Finally, (3.11) and the assumptions (3.1), (3.2), and (3.3) imply (3.12) and (3.13).

The rest of this section is devoted to the error analysis in the $L^{2}$-norm for the absolutely stabilized finite element approximation. As usual, we shall employ some appropriate duality arguments to obtain the desired results. We would like to point out that the analysis is equally applicable to the stabilized finite element formulation of Hughes and Franca [8].

Let us begin with an estimate for the pressure field $e_{p}=p-p^{h}$. Consider the following problem: find $\underset{\sim}{r}, s) \in \underset{\sim}{H_{0}^{1}}(\Omega) \times L_{0}^{2}(\Omega)$ such that

$$
\begin{cases}(\nabla \underset{\sim}{r}, \nabla \underset{\sim}{v})-(\operatorname{div} \underset{\sim}{v}, s)=0, & \underset{\sim}{v} \in{\underset{\sim}{H}}_{0}^{1}(\Omega), \\ (\operatorname{div} \underset{\sim}{r}, w)=\left(e_{p}, w\right), & w \in L_{0}^{2}(\Omega) .\end{cases}
$$

Since $e_{p} \in L_{0}^{2}(\Omega)$, it is well known that the problem (3.17) has a unique solution which satisfies the a priori estimate

$$
\|\nabla \underset{\sim}{r}\|_{0}+\|s\|_{0} \leq C\left\|e_{p}\right\|_{0}
$$

Let $\underset{\sim}{r}$ be a piecewise linear interpolation of $\underset{\sim}{r}$ such that

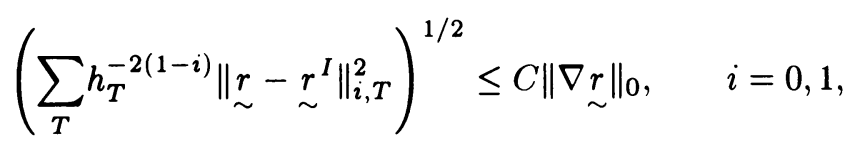

and

$$
\left(\sum_{e \in \Gamma^{h}} h_{e}^{-1} \int_{e}\left|(\underset{\sim}{r} \underset{\sim}{\underset{\sim}{r}}) \cdot \underset{\sim}{n_{e}}\right|^{2} d s\right)^{1 / 2} \leq C \| \underset{\sim}{\underset{r}{r} \|_{0} .}
$$

THEOREM 3.2. Under the assumption of the Theorem 3.1,

$$
\left\|e_{p}\right\|_{0} \leq C\left(\| \underset{\sim}{\left\|\left(\underset{\sim}{e_{u}}, e_{p}\right)\right\|}+\left(\sum_{T} h_{T}^{2}\left\|\underset{\sim}{e_{u}}\right\|_{0, T}^{2}\right)^{1 / 2}\right) .
$$


Moreover,

$$
\left\|e_{p}\right\|_{0} \leq C h^{k}\left(\|\underset{\sim}{u}\|_{k+1}+\|p\|_{k}\right)
$$

provided that $\underset{\sim}{u}, p) \in \underset{\sim}{H^{k+1}}(\Omega) \times H^{k}(\Omega)$.

Proof. It follows from (3.17) that

$$
\left\|e_{p}\right\|_{0}^{2}=\left(\operatorname{div} \underset{\sim}{r}, e_{p}\right)=\left(\operatorname{div}\left(\underset{\sim}{r}-\underset{\sim}{r}{ }^{I}\right), e_{p}\right)+\left(\operatorname{div} \underset{\sim}{r}{ }^{I}, e_{p}\right) .
$$

Since $\underset{\sim}{r}$ is a piecewise linear function, the error equation (2.16) implies that

$$
\left(\operatorname{div} \underset{\sim}{r}{ }_{\sim}^{I}, e_{p}\right)=\left(\underset{\sim}{\underset{\sim}{e}}, \nabla \underset{\sim}{r^{I}}\right)
$$

so that, by (3.18) and (3.19),

$$
\begin{aligned}
& \left|\left(\operatorname{div} \underset{\sim}{r^{I}}, e_{p}\right)\right| \leq\left\|\nabla \underset{\sim}{\underset{\sim}{e_{u}}}\right\|_{0}\left\|\nabla \underset{\sim}{\nabla}{ }^{I}\right\|_{0} \leq C\left\|\nabla \underset{\sim}{e_{u}}\right\|_{0}\|\nabla \underset{\sim}{r}\|_{0} \\
& \leq C\left\|\nabla \underset{\sim}{e_{u}}\right\|_{0}\left\|e_{p}\right\|_{0} \text {. }
\end{aligned}
$$

On the other hand, integration by parts gives

$$
\left.\left(\operatorname{div}\left(\underset{\sim}{r}-\underset{\sim}{r}{ }^{I}\right), e_{p}\right)=\sum_{T}\left(\underset{\sim}{r}-\underset{\sim}{r},-\nabla e_{p}\right)_{T}+\sum_{e \in \Gamma_{0}^{h}}\left((\underset{\sim}{r}-\underset{\sim}{r})^{I}\right) \cdot \underset{\sim}{n_{e}}, \llbracket e_{p} \rrbracket\right)_{e},
$$

since $\underset{\sim}{r}$ and $\underset{\sim}{r}$ vanish on $\partial \Omega$. Thus,

$$
\begin{aligned}
& \left.\mid\left(\operatorname{div} \underset{\sim}{r}-\underset{\sim}{r}{ }^{I}\right), e_{p}\right) \mid \leq\left(\sum_{T} h_{T}^{-2}\left\|\underset{\sim}{r}-\underset{\sim}{r}{ }^{I}\right\|_{0, T}^{2}\right)^{1 / 2}\left(\sum_{T} h_{T}^{2}\left\|\nabla e_{p}\right\|_{0, T}^{2}\right)^{1 / 2} \\
& \left.+\left.\left(\sum_{e \in \Gamma_{0}^{h}} h_{e}^{-1} \int_{e} \mid(\underset{\sim}{r}-\underset{\sim}{r})^{I}\right) \cdot \underset{\sim}{n_{e}}\right|^{2} d s\right)^{1 / 2}\left\|\llbracket e_{p} \rrbracket\right\|
\end{aligned}
$$

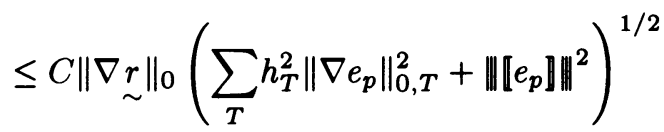

$$
\begin{aligned}
& \leq C\left\|e_{p}\right\|_{0}\left(\sum_{T} h_{T}^{2}\left\|\nabla e_{p}\right\|_{0, T}^{2}+\left\|\llbracket e_{p} \rrbracket\right\|^{2}\right)^{1 / 2},
\end{aligned}
$$

where we have used (3.19), (3.20), and (3.18). By combining (3.23) with (3.24) and (3.25), we find that

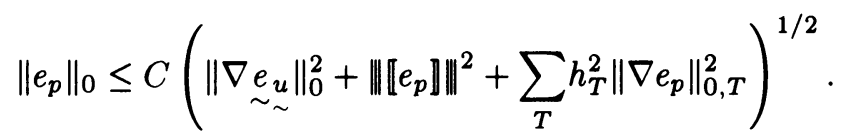

To prove (3.21), let us observe that

$$
\begin{aligned}
& \sum_{T} h_{T}^{2}\left\|\nabla e_{p}\right\|_{0, T}^{2}=\sum_{T} h_{T}^{2}\left\|\nabla e_{p}-\Delta \underset{\sim}{e_{u}}+\Delta \underset{\sim}{e_{u}}\right\|_{0, T}^{2} \\
& \leq 2\left(\sum_{T} h_{T}^{2}\left\|\nabla e_{p}-\underset{\sim}{\Delta \underset{\sim}{e}}\right\|_{0, T}^{2}+\sum_{T} h_{T}^{2}\left\|\underset{\sim}{e_{\sim}}\right\|_{0, T}^{2}\right) .
\end{aligned}
$$


Thus,

$$
\left.\left\|e_{p}\right\|_{0} \leq C\left(\| \underset{\sim}{\|\left(\sim_{\sim}^{e}\right.}, e_{p}\right) \|+\left(\sum_{T} h_{T}^{2}\left\|\underset{\sim}{e_{u}^{u}}\right\|_{0, T}^{2}\right)^{1 / 2}\right) .
$$

Finally, (3.22) is a trivial application of (3.12), (3.21), (3.13), and the standard inverse inequality.

To establish an error estimate in $L^{2}$ for the velocity field, we need to make an additional assumption on the domain $\Omega$. Let $\Omega$ be a convex polygon in $\mathbf{R}^{n}$ with $n \leq 3$. Consider the following problem: find $\underset{\sim}{q}, \theta) \in \underset{\sim}{H_{0}^{1}}(\Omega) \times L_{0}^{2}(\Omega)$ such that

$$
\begin{cases}(\underset{\sim}{\nabla}, \underset{\sim}{\nabla})-(\operatorname{div} \underset{\sim}{v}, \theta)=\left(\underset{\sim}{e_{\sim}}, \underset{\sim}{v}\right), & \underset{\sim}{v} \in \underset{\sim}{H_{0}^{1}}(\Omega), \\ (\operatorname{div} q, w)=0, & w \in L_{0}^{2}(\Omega) .\end{cases}
$$

The convexity of $\Omega$ and the $C^{0}$-smoothness of $\underset{\sim}{e_{u}}$ ensure that there exists a unique solution $(\underset{\sim}{q}, \theta) \in\left(\underset{\sim}{H^{2}}(\Omega) \cap \underset{\sim}{H}(\Omega)\right) \times\left(H^{1}(\Omega) \cap L_{0}^{2}(\Omega)\right)$ such that the following a priori estimate holds [9]:

$$
\|\underset{\sim}{q}\|_{2}+\|\theta\|_{1} \leq C \underset{\sim}{\|} \underset{\sim}{e_{u}} \|_{0}
$$

Let $\underset{\sim}{q^{I}}$ denote a $C^{0}$-piecewise linear interpolation of $\underset{\sim}{q}$, and let $\theta^{I}$ indicate either a $C^{0}$-piecewise linear or a piecewise constant interpolation of $\theta$, depending on the structure of the finite element space $M^{h}$. Assume $\underset{\sim}{\left(q^{I}, \theta^{I}\right)}$ to have the following properties:

$$
\begin{gathered}
\|\underset{\sim}{q}-\underset{\sim}{q}\|_{i} \leq C h^{2-i}\|\underset{\sim}{q}\|_{2}, \quad i=0,1 \\
\left(\sum_{T} h_{T}^{2}\left\|\nabla \theta^{I}\right\|_{0, T}^{2}\right)^{1 / 2}+\left(\sum_{T} h_{e}\|\| \theta^{I} \rrbracket \|_{0, e}^{2}\right)^{1 / 2} \leq C h\|\nabla \theta\|_{0},
\end{gathered}
$$

and

$$
\left\|\theta-\theta^{I}\right\|_{0} \leq C h\|\nabla \theta\|_{0}
$$

THEOREM 3.3. In addition to the shape regularity assumption on the triangulation $\Im_{h}$, assume that $\Omega$ is a convex polygon in $\mathbf{R}^{n}$. Then,

$$
\left\|\underset{\sim}{e_{u}}\right\|_{0} \leq C h\left(\left\|\left(\underset{\sim}{e_{u}}, e_{p}\right)\right\|+\left\|e_{p}\right\|_{0}\right)
$$

Moreover,

$$
\left\|\underset{\sim}{e_{\sim}}\right\|_{0} \leq C h^{k+1}\left(\|\underset{\sim}{u}\|_{k+1}+\|p\|_{k}\right)
$$

provided that $\underset{\sim}{u}, p) \in{\underset{\sim}{H}}^{k+1}(\Omega) \times H^{k}(\Omega)$.

Proof. By (3.27),

$$
\left\|\underset{\sim}{e_{u}}\right\|_{0}^{2}=\left(\nabla \underset{\sim}{q}, \nabla \underset{\sim}{\nabla} \underset{\sim}{e_{u}}\right)-\left(\operatorname{div} \underset{\sim}{e_{u}^{u}}, \theta\right)
$$

Set

$$
A=\left(\underset{\sim}{q}, \underset{\sim}{\nabla} \underset{\sim}{e_{u}}\right)
$$


and

$$
B=\left(\operatorname{div} \underset{\sim}{\underset{\sim}{e_{u}}}, \theta\right)=\left(\operatorname{div} \underset{\sim \sim \sim}{e_{u}}, \theta-\theta^{I}\right)+\left(\operatorname{div} \underset{\sim}{\underset{\sim}{u}}, \theta^{I}\right) .
$$

Since $\underset{\sim}{q^{I}} \in X^{h}$ is a piecewise-linear function, the error equation (2.16) implies that

$$
\begin{aligned}
A & =\left(\nabla\left(\underset{\sim}{q}-{\underset{\sim}{q}}^{I}\right), \nabla \underset{\sim}{\nabla} e_{\sim}\right)+\left(\nabla \underset{\sim}{\nabla}{ }_{\sim}^{I}, \nabla \underset{\sim}{\nabla}{\underset{\sim}{u}}_{u}\right) \\
& =\left(\nabla\left(\underset{\sim}{q}-{\underset{\sim}{q}}^{I}\right), \nabla \underset{\sim}{e_{u}}\right)+\left(\operatorname{div}\left({\underset{\sim}{q}}^{I}-\underset{\sim}{q}\right), e_{p}\right) .
\end{aligned}
$$

Hence,

$$
\begin{aligned}
|A| & \leq\left\|\nabla\left(\underset{\sim}{q-\sim_{\sim}^{q}}\right)\right\|_{0}\left(\left\|\nabla \underset{\sim}{e_{u}}\right\|_{0}+\left\|e_{p}\right\|_{0}\right) \\
& \leq C h\left\|\underset{\sim}{e_{u}}\right\|_{0}\left(\left\|\nabla \underset{\sim}{e_{\sim}}\right\|_{0}+\left\|e_{p}\right\|_{0}\right),
\end{aligned}
$$

where we have used (3.29) and (3.28) in deriving (3.38).

Next,

$$
\begin{aligned}
|B| & \leq\|\nabla \underset{\sim}{\underset{\sim}{u}}\|_{0}\left\|\theta-\theta^{I}\right\|_{0}+\left|\left(\operatorname{div} \underset{\sim}{\underset{\sim}{e}}, \theta^{I}\right)\right| \\
& \leq C h\left\|\nabla \underset{\sim}{e_{\sim}}\right\|\left\|_{0}\right\| \theta \|_{1}+\left|\left(\operatorname{div} \underset{\sim}{e_{\sim}^{u}}, \theta^{I}\right)\right| \\
& \leq C h\left\|\nabla \underset{\sim}{e_{\sim}^{u}}\right\|_{0}\left\|{\underset{\sim}{u}}_{\sim}^{e_{u}}\right\|_{0}+\left|\left(\operatorname{div} \underset{\sim}{e_{u}^{u}}, \theta^{I}\right)\right| .
\end{aligned}
$$

Clearly, it suffices to bound

$$
D=\left(\operatorname{div} \underset{\sim}{\underset{\sim}{e}}{ }_{\sim}, \theta^{I}\right) .
$$

In fact, the error equation (2.16) implies that

$$
D=-\sum_{T} h_{T}^{2}\left(\nabla e_{p}-\Delta \underset{\sim}{e_{u}^{u}}, \nabla \theta^{I}\right)_{T}-\sum_{e \in \Gamma_{0}^{h}} h_{e}\left(\llbracket e_{p} \rrbracket,\left[\theta^{I}\right]\right)_{e}
$$

Thus,

$$
\begin{aligned}
|D| \leq & \left(\sum_{T} h_{T}^{2}\left\|\nabla e_{p}-\underset{\sim}{\underset{\sim}{e_{u}}}\right\|_{0, T}^{2}\right)^{1 / 2}\left(\sum_{T} h_{T}^{2}\left\|\nabla \theta^{I}\right\|_{0, T}^{2}\right)^{1 / 2} \\
& \left.+\left\|\llbracket e_{p}\right\|\left\|_{0, \Gamma_{0}^{h}}\right\| \llbracket \theta^{I}\right] \|_{0, \Gamma_{0}^{h}} .
\end{aligned}
$$

Furthermore, an application of (3.30) and (3.31) to (3.41) leads to

$$
|D| \leq C h\|\nabla \theta\|_{0}\left(\left(\sum_{T} h_{T}^{2}\left\|\nabla e_{p}-\underset{\sim}{e_{u}}\right\|_{0, T}^{2}\right)^{1 / 2}+\left\|\llbracket e_{p}\right\|_{0, \Gamma_{0}^{h}}\right) .
$$

Thus, combining (3.39) with (3.42) and using (3.28), gives

$$
|B| \leq C h\left\|\left(\underset{\sim}{e_{u}}, e_{p}\right)\right\|\left\|\underset{\sim}{e_{u}}\right\|_{0}
$$

Now, (3.32) follows from (3.34) combined with (3.38) and (3.43).

Department of Mathematics

Purdue University

West Lafayette, Indiana 47907

Department of Mathematics

The University of Chicago

Chicago, Illinois 60637 
1. I. BABUŠKA, "The finite element method with Lagrangian multipliers," Numer. Math., v. 20, 1973, pp. 179-192.

2. F. BREZZI, "On the existence, uniqueness, and approximation of saddle point problems arising from Lagrangian multipliers," RAIRO Anal. Numér., v. 2, 1974, pp. 129-151.

3. F. BREzzI \& J. Douglas, JR., "Stabilized mixed method for the Stokes problem," Numer. Math., v. 53, 1988, pp. 225-235.

4. F. BREZZI \& J. PITKÄRANTA, "On the stabilization of finite element approximations of the Stokes equations," Efficient Solutions of Elliptic Systems, Notes on Numerical Fluid Mechanics, Vol. 10 (W. Hackbusch, ed.), Vieweg, Braunschweig/Wiesbaden, 1984.

5. R. S. FALK \& J. E. OSBORN, "Error estimates for mixed methods," RAIRO Anal. Numér., v. 14,1980 , pp. $249-277$.

6. V. GiRAUlt \& P. A. RAVIART, Finite Element Methods for Navier-Stokes Equations, SpringerVerlag, Berlin-Heidelberg, 1986.

7. T. J. R. HUGheS, L. P. FRANCA \& M. BALESTRA, "A new finite element formulation for computational fluid dynamics: V. Circumventing the Babuska-Brezzi condition: A stable PetrovGalerkin formulation of the Stokes problem accommodating equal-order interpolation," Comput. Methods Appl. Mech. Engrg., v. 59, 1986, pp. 85-99.

8. T. J. R. HUGHes \& L. P. FRANCA, "A new finite element formulation for computational fluid dynamics: VII. The Stokes problem with various well-posed boundary conditions: symmetric formulations that converge for all velocity/pressure spaces," Comput. Methods Appl. Mech. Engrg., v. 65, 1987, pp. 85-96.

9. R. B. KELlogG \& J. E. OSBORN, "A regularity result for the Stokes problem in a convex polygon," J. Funct. Anal., v. 21, 1976, pp. 397-431. 